eISSN 2444-7986

DOI: http://dx.doi.org/10.14201/orl201673.14912

\title{
Editorial
}

\section{REVISTAS DE OTORRINOLARINGOLOGÍA (ORL): IMPACTO Y VISIBILIDAD}

\author{
ORL Journals: impact and visibility
}

\author{
Helena MARTÍN-RODERO \\ Universidad de Salamanca. Facultad de Medicina. Biblioteca. Revista ORL. Consejo Editorial. Salamanca. España. \\ http://orcid.org/0000-0002-6698-9240 \\ Correspondencia: helena@usal.es
}

Fecha de Publicación: 22 de julio de 2016

Conflicto de intereses: Los autores declaran no tener conflictos de intereses

Imágenes: Los autores declaran haber obtenido las imágenes con el permiso de los pacientes

Política de derechos y autoarchivo: se permite el autoarchivo de la versión post-print (SHERPA/RoMEO)

Licencia CC BY-NC-ND. Licencia Creative Commons Atribución-NoComercial-SinDerivar 4.0 Internacional

๔ Universidad de Salamanca. Su comercialización está sujeta al permiso del editor

El pasado mes de junio, como cada año, hemos conocido el Factor de Impacto calculado por el Journal Citation Reports (JCR) de Thomson Reuters para el año 2015.

Quizá sea un buen momento para analizar, de una forma descriptiva, cuál es el impacto y la visibilidad de las revistas de Otorrinolaringología a través de su presencia en el JCR y MEDLINE.

El Factor de Impacto (FI) es considerado por algunos autores como un indicador indirecto de la calidad, prestigio y visibilidad de las publicaciones científicas [1]. A pesar de las opiniones críticas vertidas sobre su utilidad para valorar la actividad investigadora, de sus limitaciones y sesgos [2-6], el FI sigue siendo uno de los indicadores más utilizados por investigadores, editores y agencias de evaluación y financiación. El Journal Citation Reports es publicado anualmente por
Thomson Reuters y está disponible para las instituciones académicas y de investigación españolas gracias a una licencia nacional suscrita por la Fundación Española de Ciencia y Tecnología (FECyT) https://www.recursoscientificos.fecyt.es/-.

MEDLINE, por su parte, es considerada, no sólo una de las mejores bases de datos de literatura biomédica, sino un instrumento de evaluación de la calidad de las revistas biomédicas, debido, entre otras razones, a la rigurosa selección que la National Library of Medicine (NLM) hace de las publicaciones indizadas en sus bases de datos. La NLM dispone de un comité de expertos (Literature Selection Technical Review Committee, LSTRC) encargado de seleccionar las mejores publicaciones, atendiendo a criterios como ámbito, cobertura, audiencia, calidad del contenido y calidad editorial de las mismas [7]. 
REVISTAS DE OTORRINOLARINGOLOGÍA

Analizando los datos que nos ofrece el Journal Citation Reports para el año 2015 vemos que el número total de revistas incluidas para todas las categorías ha sido de 11.990 , de las cuales 8.778 pertenecen a la sección Science Citation Index Expanded (SCIE) y 3.212 a la sección Social Science Citation Index (SSCI). El número de revistas pertenecientes a la categoría "Otorhinolaryngology» del SCIE es de 43 , un $0,49 \%$ del total de revistas pertenecientes al SCIE, si lo comparamos con las 29 revistas presentes en el JCR en el año 2005, vemos que ha habido un incremento medio anual del 1,4 .

La revista con un Factor de Impacto más alto en 2015 ha sido Hearing Research con un FI de 3,565 mientras que la revista con un Factor de Impacto más bajo ha sido Journal of International Advanced Otology con un FI de 0,108 (Tabla 1).

En cuanto al número de artículos publicados, la revista más prolífica ha sido Laryngoscope con un total de 549 artículos, lo que supone un $9,54 \%$ del total de artículos publicados en la categoría, siendo también esta revista la más citada por las revistas incluidas en el JCR para el año analizado, recibiendo un total de 19.229 citas $(14,78 \%)$ (Tabla 1$)$.

Por lo que respecta a la distribución por países, es Estados Unidos el país que más revistas de la especialidad aporta al JCR con un total de 22 revistas $(51,16 \%)$, le sigue los Países Bajos con $4(9,30 \%)$, Reino Unido y Alemania con $3(6,98 \%)$, Canadá y Suiza con $2(4,65 \%)$. El resto de países, Italia, Francia, Corea del Sur, Noruega, Brasil y Turquía están presentes con 1 publicación $(2,33 \%)$. Por último, señalar que no encontramos ninguna revista española de la especialidad en el JCR (Tabla 1).

El inglés es el idioma predominante.

Igualmente, se ha analizado la presencia de las revistas de Otorrinolaringología en la base de datos MEDLINE, a través de PubMed http://www.pubmed.gov-. Para ello, se realizó una búsqueda en el Journal In NCBI Databases

http://www.ncbi.nlm.nih.gov/nlmcatalog/journa Is - mediante la siguiente estrategia de búsqueda [("Otolaryngology"[MeSH] OR "Otolaryngology"[AIl Field] OR "Otorhinolaryngology"[All Field]) AND "periodicals"[publication type] AND ncbijournals[All Fields]] con el fin de identificar las revistas, en curso, de la especialidad y posteriormente buscar aquellas que estaban indexadas en la base de datos MEDLINE, para ello se completó la sentencia anterior con el filtro [currentlyindexed[AII]. El número de revistas, en curso, presentes en el catálogo de publicaciones periódicas de la NLM, National Library of Medicine (EE.UU.) fue de 69 de las cuales 44 están indexadas en MEDLINE y de éstas, un $68,18 \%$ tienen Factor de Impacto asociado. El predominio sigue siendo de Estados Unidos y de las publicaciones en inglés. Hay que aclarar que no existe una correspondencia exacta entre las categorías temáticas del JCR y MEDLINE. De esta manera. analizando las revistas de la categoría "Otorhinolaryngology» del JCR vemos que tan sólo 2 revistas no están indexadas en MEDLINE (Clinical and Experimental Otorhinolaryglogy y Trends in Amplification, ésta última cesada en 2015 y continuada por Trends Hearing). El resto de las revistas sí lo están, un $69,77 \%$ bajo la categoría «Otolaryngology» y el $25,58 \%$ restante bajo otras categorías temáticas como "Audiology» o «Hearing Disorders».

En cuanto a la presencia de revistas españolas de Otorrinolaringología en bases de datos internacionales vemos que es meramente testimonial. En España, en el momento actual, tan solo se publican 4 revistas de ORL, todas pertenecientes a sociedades científicas, a saber: Acta Otorrinolaringológica Española, Acta Otorrinolaringológica Gallega, ORL Aragón y, finalmente, Revista ORL. Acta Otorrinolaringológica Española es, sin duda, la más internacional de todas, siendo indexada en las principales bases de datos: MEDLINE, Scopus, o Web of Science.

A la vista de la situación, podemos concluir que a nivel internacional las revistas de ORL experimentan un crecimiento sostenido con un predominio de Estados Unidos y de las publicaciones en lengua inglesa.

En relación a las revistas españolas de ORL, se observa que todavía tienen un largo recorrido por delante y han de superar las limitaciones impuestas por el tamaño del área disciplinar con un número reducido de revistas científicas, centradas en temas locales y orientadas a la investigación clínica o aplicada y en menor medida a la investigación básica, hecho que, como apunta Bordons et al. [1] se asocia, independientemente de la calidad de las revistas, con una difusión más restringida que dificulta la indexación en bases de datos internacionales, la citación y en consecuencia su indexación en los índices de im- 
pacto. Por otro lado, no hay que pasar por alto la todavía reducida presencia de revistas españolas en el Journal Citation Reports, hecho que refleja, como ya han apuntado distintos autores, el evidente sesgo que presenta esta base de datos a favor de las publicaciones anglosajonas.

Tabla 1. Revistas de la categoría «Otorhinolaryngology» según el Journal Citation Reports, 2015.

\begin{tabular}{|c|c|c|c|c|c|}
\hline $\mathrm{N}^{\circ}$ & Título abreviado* & $\mathrm{FI}$ & $\mathrm{N}^{\circ}$ art. & Citas totales & País \\
\hline 1 & Hear Res & 3.565 & 147 & 8.627 & 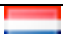 \\
\hline 2 & J Assoc Res Otolaryngol & 3.030 & 53 & 2.044 & 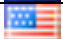 \\
\hline 3 & Head Neck & 2.760 & 303 & 8.900 & 溧悲 \\
\hline 4 & JAMA Otolaryngol Head Neck Surg & 2.705 & 137 & 891 & 跙春 \\
\hline 5 & Clin Otolaryngol & 2.627 & 74 & 2.435 & 运 \\
\hline 6 & Ear Hear & 2.517 & 111 & 4.467 & 嚂 \\
\hline 7 & Trends Amplif** & 2.500 & 0 & 480 & 㗐真 \\
\hline 8 & Int Forum Allergy Rhinol & 2.350 & 184 & 1.338 & 㗼春 \\
\hline 9 & Laryngoscope & 2.272 & 549 & 19.229 & 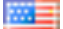 \\
\hline 10 & Trends Hear & 2.158 & 25 & 69 & 監亘 \\
\hline 11 & Otolaryngol Head Neck Surg & 2.021 & 336 & 11.355 & 㗐刲 \\
\hline 12 & Am J Rhinol Allergy & 1.960 & 119 & 3.217 & 嚂 \\
\hline 13 & Otol Neurotol & 1.953 & 302 & 6.237 & 覀羊 \\
\hline 14 & Audiol Neuro Otol & 1.776 & 61 & 1.646 & 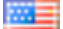 \\
\hline 15 & Dysphagia & 1.754 & 67 & 2.231 & 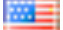 \\
\hline 16 & Rhinology & 1.686 & 52 & 2.018 & \\
\hline 17 & Int J Audiol & 1.681 & 140 & 2.689 & \\
\hline 18 & Eur Arch Otorhinolaryngol & 1.627 & 493 & 5.567 & \\
\hline 19 & Curr Opin Otolaryngol Head Neck Surg & 1.586 & 78 & 1.744 & 監 \\
\hline 20 & J Otolaryngol Head Neck Surg & 1.585 & 56 & 1.610 & t \\
\hline 21 & Acta Otorhinolaryngol Ital & 1.531 & 72 & 1.069 & \\
\hline 22 & J Am Acad Audiol & 1.344 & 71 & 1.798 & \\
\hline 23 & Otolaryngol Clin North Am & 1.264 & 76 & 2.215 & 㗐㭅 \\
\hline 24 & Ann Otol Rhinol Laryngol & 1.171 & 169 & 5.890 & 監 \\
\hline 25 & Acta Oto Laryngol & 1.127 & 207 & 6.254 & \\
\hline 26 & Int J Pediatr Otorhinolaryngol & 1.125 & 481 & 5.619 & \\
\hline 27 & Am J Audiol & 1.125 & 63 & 493 & 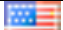 \\
\hline 28 & J Voice & 1.113 & 144 & 3.053 & 監具 \\
\hline 29 & J Vestib Res Equilib Orientat & 1.047 & 21 & 829 & 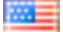 \\
\hline 30 & Auris Nasus Larynx & 1.038 & 99 & 1.522 & \\
\hline 31 & J Otorhinolaryngol Head Neck Surg & 1.000 & 44 & 1.057 & {$[3$} \\
\hline 32 & Eur Ann Otorhinolaryngol Head Neck Dis & 0.942 & 55 & 357 & \\
\hline 33 & Am J Otolaryngol & 0.933 & 168 & 2.216 & 盜具 \\
\hline 34 & Ear Nose Throat J & 0.932 & 49 & 1.520 & 潭春 \\
\hline 35 & Clin Exp Otorhinolaryngol & 0.855 & 69 & 384 & 8 \\
\hline 36 & HNO & 0.852 & 96 & 1.095 & \\
\hline 37 & Logop Phoniatr Vocology & 0.750 & 23 & 341 & \\
\hline 38 & J Laryngol Otol & 0.736 & 274 & 4.847 & 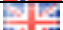 \\
\hline 39 & Braz J Otorhinolaryngol & 0.730 & 91 & 869 & 2 \\
\hline 40 & Laryngo-Rhino-Otol & 0.572 & 85 & 776 & 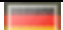 \\
\hline 41 & B-ENT & 0.487 & 63 & 276 & $\Gamma$ \\
\hline 42 & Folia Phoniat. Logop & 0.391 & 11 & 737 & {$[3$} \\
\hline 43 & J Int Adv Otol & 0.108 & 39 & 54 & $c_{t}$ \\
\hline
\end{tabular}


La estrategia adoptada por Revista ORL, de difusión en abierto, con un claro compromiso por la internacionalización, la calidad formal y de contenidos abre nuevas posibilidades para una mayor difusión, visibilidad y quién sabe si, en un futuro no muy lejano, también para el impacto.

\section{BIBLIOGRAFÍA}

1. Bordons M, Felipe A, Gómez I. Revistas científicas españolas con Factor de Impacto en el año 2000. Rev Esp Doc Cient 2002;25(1):49-73.

2. Seglen PO. Why the impact factor of journals should not be used for evaluating research. BMJ [Internet] 1997 [citado 2016 jul 21];314(7079):498-502. Disponible en: http://www.ncbi.nlm.nih.gov/pmc/article s/PMC2126010/.

3. Kurmis AP. Understanding the limitations of the journal impact factor. J Bone Joint Surg Am 2003;85-A(12):2449-54.

4. Bordons M, Zulueta MÁ. Evaluación de la actividad científica a través de indicadores bibliométricos. Rev Esp Car- diol [Internet] 1999 [citado 2016 jul 12];52(10):790-800. Disponible en: http://www.revespcardiol.org/es/evaluac ion-actividad-cientifica-travesindicadores/articulo/190/.

5. Aleixandre-Benavent $\mathrm{R}$, ValderramaZurián JC, González-Alcaide G. El factor de impacto de las revistas científicas: limitaciones e indicadores alternativos. El Prof Inf [Internet] 2007[citado 2016 jul 21];16(1):4-12. Disponible en: https://dialnet.unirioja.es/servlet/articulo ?codigo $=2224830$.

6. Rodríguez Martín JL. El Factor de Impacto como medida de relevancia en la Investigación Clínica. Rev Clínica Med Fam [Internet] 2008 [citado 2016 jul 21];2(3):97-8. Disponible en: http://www.redalyc.org/articulo.oa? $i d=1$ 69614507001.

7. Fact SheetMEDLINE® Journal Selection [Internet]. US Natl. Libr. Med. Internte Bethesda US Natl. Libr. Med.1988 [citado 2014 mar 18];Disponible en: http://www.nlm.nih.gov/pubs/factsheets/ jsel.html. 